\title{
SIMULATING THE SOLAR DYNAMO
}

\author{
AXEL BRANDENBURG \\ HAO/NCAR* , P.O. Box 3000, Boulder, CO 80307, USA
}

\begin{abstract}
Mean-field and direct simulations of the hydrodynamics and hydromagnetics of the solar convection zone are discussed with the ultimate aim to understand the generation of differential rotation and magnetic fields. Various arguments constraining the values of the various turbulent diffusion coefficients are presented. It is suggested that the turbulent magnetic diffusivity is much smaller than the eddy viscosity which, in turn, is by up to a factor of ten smaller than the eddy conductivity. The magnetic field obtained from direct simulations is highly intermittent, and there is no clear systematic orientation of bipolar regions emerging from the convection zone. Various mechanisms that might cause such a field orientation are considered. Finally, the application of direct simulations to the determination of mean-field transport coefficients is emphasised.
\end{abstract}

Key words: Solar dynamo - mean-field dynamo - direct simulation

\section{Introduction}

Solving the entire solar dynamo on the computer still remains an intractable problem. Nevertheless, with reasonable approximations we may hope to model more realistically the solar dynamo within the not too far distant future. The pioneering approach of Gilman and Glatzmaier about ten years ago was an example of treating the relevant physics explicitly (Gilman \& Miller 1981, Gilman 1983, Glatzmaier 1985a). No explicit $\alpha$-effect was assumed and the magnetic field was generated solely by the large-scale convective motions. However, a small-scale turbulent heat transport was modelled by including an additional small-scale convective flux term, and the conductivity and viscosity coefficients were fairly large $\left(Z 10^{12} \mathrm{~cm}^{2} / \mathrm{s}\right)$.

Up to now it is not entirely clear why this promising approach did not produce correctly some of the essential properties of the solar dynamo such as the equatorward migration of sunspot regions (butterfly diagram), and a rotation law compatible with helioseismology (e.g. Brown \& Morrow 1987). There are some suggestions that the dynamo should instead be placed in the overshoot region at the bottom of the convection zone (Glatzmaier $1985 \mathrm{~b}$, see also the recent review by Gilman 1992). It has also been proposed that the physics of small-scale motions at the scale of granulation should be treated explicitly in the model (Nordlund 1985). Meanwhile we know that highly concentrated downdrafts can extend over several pressure scale heights (Stein \& Nordlund 1989), and may persist throughout a major part of the solar convection zone. It would indeed be surprising if such a scenario could be successfully approximated by computing only large-scale convective motions. However, it still needs to be demonstrated that the presence of these concentrated downdrafts will really solve the basic problems.

The possible importance of resolving small-scale convective motions in a dynamo motivates the restriction to a small Cartesian subvolume located somewhere in the lower part of the convection zone. Direct simulations of convective dynamo action have demonstrated that strong and irregular small-scale magnetic fields are generated at sufficiently large magnetic Reynolds numbers (Meneguzzi \& Pouquet

* The National Center for Atmospheric Research is sponsored by the National Science Foundation 
1989, Nordlund et al. 1992). This may have consequences for the traditional concept of an $\alpha$-effect and the turbulent diffusivity. Conventional $\alpha$-effect dynamos ignore the effects of small-scale magnetic fluctuations and the computation of the $\alpha$-effect is based entirely on the small-scale velocity field (e.g. Krause \& Rädler 1980). It is possible, however, that the large-scale solar dynamo works in an environment with a strong background magnetic field and the small-scale magnetic fields then play an equally important role to the small-scale velocity field that is usually considered to derive turbulent transport coefficients. In fact, there are examples where a turbulent diamagnetic effect has been derived that does include the effects of a small-scale magnetic field (Kichatinov 1991). A suppression of the $\alpha$-effect by the magnetic current helicity, $\left\langle\mathbf{J}^{\prime} \cdot \mathbf{B}^{\prime}\right\rangle$, has been proposed in an early paper by Vainshtein (1972). The importance of the small-scale magnetic field on the strength of $\alpha$ and $\eta$ quenching has recently been stressed by Vainshtein \& Cattaneo (1992). This issue will be discussed in more detail below.

We begin by summarising some recent results and implications of hydromagnetic mean-field dynamos. After this we review some results of direct simulations of dynamo action and discuss how such simulations can be used to measure turbulent transport coefficients. Finally the role of the mean magnetic field in a direct simulation is discussed and we speculate how the systematic orientation of bipolar regions and sunspot pairs can be explained.

\section{The mean-field dynamo problem}

\section{1. $\alpha \Omega$-DYNAMOS}

There have been several attempts to improve the mean-field dynamo approach and to apply it to the Sun. Nevertheless, many models do not reproduce important geometrical properties of the solar magnetic field. In fact, the theoretical butterfly diagram of the old Model I of Steenbeck \& Krause (1969) is often better than many butterfly diagrams presented since. The main "problem" arose with the better knowledge of the solar internal angular velocity distribution. Models with an inward increasing angular velocity in the equatorial plane (i.e. $\partial \Omega / \partial r<0$ ), as in the models of Steenbeck \& Krause, unfortunately do not apply to the Sun.

Within the framework of $\alpha \Omega$-dynamos an equatorward migration of the sunspot belts can be obtained when $\alpha \partial \Omega / \partial r$ is positive in the northern hemisphere. Attempts to construct a solar dynamo with $\partial \Omega / \partial r>0$ and negative $\alpha$-effect (in the northern hemisphere) have been reviewed by Schmitt (1993). In this approach, the two induction effects, differential rotation and $\alpha$-effect, are placed in the overshoot layer at the bottom of the solar convection zone. This is in contrast to the original ideas of Steenbeck and Krause, where the two induction effects operate in spatially distinct regions. It is still not clear, however, which of the two scenarios is actually more appropriate to the Sun. In a recent paper by Parker (1993) the idea of distinct regions of induction effects has been reconsidered in a model for the interface between the convection zone proper and the radiative interior.

A realistic model of the solar dynamo should indeed include the combined system of convection zone and overshoot layer. This can be modelled by including a 
layer with reduced turbulent diffusion coefficients below the convection zone proper. Oscillatory magnetic fields generated in the convection zone only penetrate of order a skin depth into the interior, as demonstrated by Roberts \& Stix (1972). However, if a downward turbulent pumping is included the field can accumulate near the interface. Some preliminary models of this kind have been presented in Brandenburg et al. (1992a), where the equations describing the generation of differential rotation by the $\Lambda$-effect have been solved simultaneously with the $\alpha$-effect dynamo equations. This more consistent approach reduces the degree of freedom of setting up such models. In the following we summarise the basic constraints that arise when the equations for the fluid motions are solved simultaneously.

\subsection{Differential Rotation}

Nonlinear models for the solar differential rotation as well as direct simulations of convection in rotating spherical shells (e.g. Glatzmaier \& Gilman 1982) often tend to produce $\Omega$-contours that are constant on cylinders. This contradicts current results of helioseismology (e.g. Brown \& Morrow 1987, Libbrecht 1988) which suggest that the $\Omega$-contours are more radial. Recently, Gough et al. (1992) pointed out that models with cylindrical $\Omega$-contours for $\varpi>0.7 R$, where $\varpi$ is the distance from the rotation axis and $R$ the outer radius, might also be compatible with the truncated set of helioseismological data. With the definition $\mathrm{Ta}=4 \Omega^{2} R^{4} / \nu_{t}^{2}$, where $\nu_{t}$ is the eddy viscosity, we typically find that the $\Omega$-contours become cylindrical if Ta is of the order of $10^{6}$ or larger. This is demonstrated in Fig. 1, where we show contours of constant angular velocity and streamlines for compressible and stratified mean-field models with different values of Ta. In these models the angular velocity is driven by the $\Lambda$-effect, quantified by a set of parameters that are thought to be relevant for the Sun $\left[V^{(0)}=-1, V^{(1)}=H^{(1)}=5 / 4\right.$, see Rüdiger \& Tuominen (1990)].

For a small Taylor number the $\Omega$-contours are similar to those suggested by helioseismology. For a larger value of Ta the $\Omega$-contours becomes more nearly cylindrical, at least in the deeper parts of the convection zone. In the two rightmost panels a case with anisotropic eddy-conductivity is shown (see Brandenburg et al. $1992 \mathrm{~b})$. Note that the effect on the $\Omega$-contours is only modest - even for rather strong anisotropy. With stronger anisotropies noticeable deviations from cylindrical $\Omega$-contours are possible, but the resulting latitudinal convective fluxes are rather large (see Fig. 10 in Brandenburg et al. 1992b). The distribution of the convective flux at the outer radius, however, has become more radial and would therefore not necessarily contradict the observations (Spruit 1977, Durney \& Spruit 1979). The importance of a significant latitudinal dependence of the specific entropy for causing deviations from cylindrical $\Omega$-contours has been stressed by Durney (1987).

The meridional velocities generated in the case of large Taylor numbers are maximal deep in the convection zone. The surface values are much smaller and possibly close to the detectable value. 

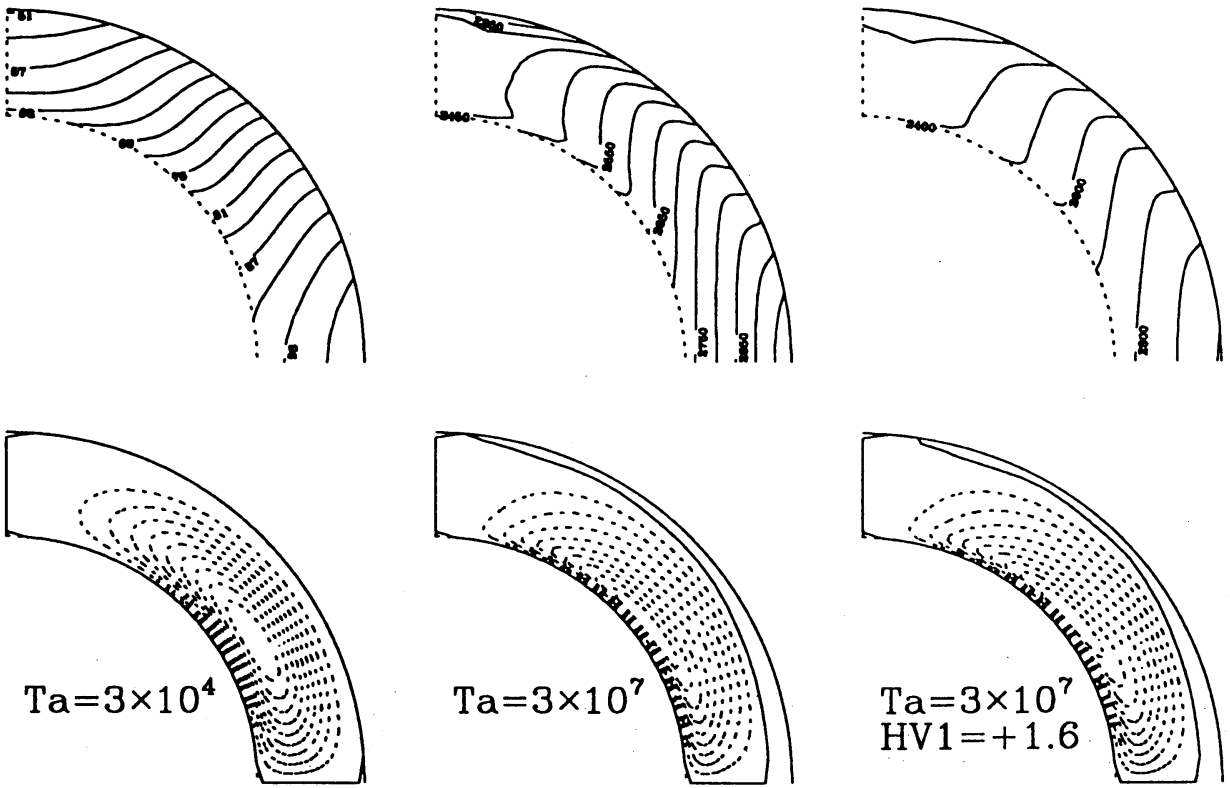

Fig. 1. Comparison of the contours of the angular velocity (upper row) and streamlines of meridional circulation (lower row) for two different values of the Taylor number and isotropic eddy conductivity (left and middle column), and anisotropic eddy conductivity (right column). Dotted streamlines denote counter-clockwise circulation.

\subsection{Constraints on the turbulent diffusion COEFFicients}

The theoretical results for the various turbulent transport coefficients are rather uncertain, and this has lead to the practice of treating them essentially as free parameters. In the following we point out that there are several constraints on the values of the various diffusion coefficients.

In the previous section we have seen that the solar differential rotation can readily be explained in terms of the $\Lambda$-effect formalism when $\mathrm{Ta} \lesssim 10^{5}$, i.e. $\nu_{t} \gtrsim$ $10^{14} \mathrm{~cm}^{2} / \mathrm{s}$. Furthermore, if we accept the idea of an $\alpha \Omega$-type dynamo as explanation for the solar cycle then, in order to obtain the correct 22 year period, the turbulent magnetic diffusivity, $\eta_{t}$, should not exceed a value around $10^{10 \ldots 11} \mathrm{~cm}^{2} / \mathrm{s}$ (e.g. Choudhuri 1990).

There are two other effects that constrain the diffusion coefficients. The equations governing the mean stratification of the convection zone may exhibit a largescale Rayleigh-Bénard type instability if the "turbulent" Rayleigh number

$$
\mathrm{Ra}_{t}=\frac{g d^{4}}{\nu_{t} \chi_{t}}\left(-\frac{1}{c_{p}} \frac{d S}{d r}\right)_{\text {mid }}=\frac{g d^{4}}{\nu_{t} \chi_{t}^{2}}\left(\frac{\nabla_{\mathrm{ad}} F_{\text {conv }}}{p}\right)_{\text {mid }},
$$

is large enough. Here, $\chi_{t}$ is the eddy conductivity, $\mathbf{F}_{\text {conv }}=-\chi_{t} \rho T \nabla S$ is the smallscale convective flux, and a perfect gas with $p=\nabla_{\mathrm{ad}} \rho c_{p} T$ has been assumed with 
$\nabla_{\mathrm{ad}}=1-1 / \gamma=0.4$. For solar values we have $\mathrm{Ra}_{t} \approx 2 \times 10^{4}$, which is somewhat above the critical value $\left(0.5 \ldots 1 \times 10^{4}\right)$ for the onset of Rayleigh-Bénard convection. A large-scale convection in addition to the small-scale convection modelled in the mean-field approach may be an artifact and an indication that the mean-field equations become invalid, or are too simple; see Brandenburg et al. (1992b) and Tuominen et al. (1993). This can be avoided by taking much larger values for $\nu_{t}$ and $\chi_{t}$, i.e. $\left(\nu_{t} \chi_{t}\right)^{1 / 2} \gtrsim 1.5 \times 10^{13} \mathrm{~cm}^{2} / \mathrm{s}$. However, there is yet another issue that, if correctly interpreted, may prevent us from choosing $\nu_{t}$ too large.

In all mean-field investigations of the solar differential rotation the turbulent viscous heating in the mean-field energy equation has been neglected. In fact, Hewitt et al. (1975) have shown that viscous heating in convective flows can contribute up to about $5 \%$ to the energy budget if the stratification is strong. If we adopt the molecular (laminar) expression for the viscous heating term with ordinary viscosity being replaced by the turbulent value, then we have $q_{\text {visc }}=-Q_{i j}\left\langle u_{i, j}\right\rangle$, where $Q_{i j}=\left\langle u_{i}^{\prime} u_{j}^{\prime}\right\rangle=-\nu_{t}\left(\left\langle u_{i, j}\right\rangle+\left\langle u_{j, i}\right\rangle\right)+\Lambda$-effect. The largest contribution comes from the differential rotation, i.e.

$$
q_{\text {visc }} \approx \rho \nu_{t}(\varpi \nabla \Omega)^{2} \text {. }
$$

In the Sun $|\varpi \nabla \Omega|$ is about $0.2 \Omega$. The integral of $q_{\text {visc }}$ over the entire convection zone leads to the estimate

$$
Q_{\mathrm{visc}} \equiv \int_{\mathrm{CZ}} q_{\mathrm{visc}} d V \approx M_{c} \nu_{t}(0.2 \Omega)^{2} \text {, }
$$

where $M_{c} \approx 6 \times 10^{31} \mathrm{~g}$ is the mass of the solar convection zone. Comparing $Q_{\text {visc }}$ with the solar luminosity we find

$$
Q_{\text {visc }} / L \approx 0.06\left(\nu_{t} / 10^{13} \mathrm{~cm}^{2} / \mathrm{s}\right) \text {. }
$$

Thus, the rate of viscous heat production can be a substantial fraction of the solar luminosity if $\nu_{t}$ is of the order of $10^{13} \mathrm{~cm}^{2} / \mathrm{s}$ or larger. We computed a number of models for different Taylor numbers with the viscous heat term included and found in these cases that the models become more unstable to convection than before. This is due to the fact that the viscous heating, since it is proportional to the density $\rho$, is more efficient in the lower parts and therefore enhances the radial entropy gradient.

Rüdiger (1987) pointed out that, in general, the coefficient in the viscous heating expression differs from the turbulent viscosity coefficient. Using the First-OrderSmoothing approximation he found that, in the context of accretion disc theory, this coefficient can be three times larger than the turbulent viscosity. This would make the viscous heating problem even more severe. Thus, if Eq. (4) is correct, we must conclude that $\nu_{t} \ll 10^{13} \mathrm{~cm}^{2} / \mathrm{s}$.

To summarise this subsection, there are arguments that in the solar convection zone the various turbulent diffusion coefficients differ from each other. Problems concerning the dynamo period indicate that $\eta_{t} \approx 10^{11} \mathrm{~cm}^{2} / \mathrm{s}$ or even smaller, whereas the possibility of large-scale Rayleigh-Bénard type convection suggests that $\chi_{t} \approx 10^{14} \mathrm{~cm}^{2} / \mathrm{s}$. The value of $\nu_{t}$ must be somewhere in between these two values, i.e., 
$\eta_{t} \ll \nu_{t} \ll \chi_{t}$.

Arguments concerning the turbulent heating and the Taylor number puzzle are not compatible with each other. If we accept the expression for the turbulent heating, then the constraint of not too large viscous heating requires $\nu_{t} \lesssim 3 \times 10^{12} \mathrm{~cm}^{2} / \mathrm{s}$. It should be noted that the ordering in Eq. (5) is not implausible and may be related to the increasing intermittency of entropy, velocity, and magnetic field structures.

\section{Intermittency and magnetic field orientation}

Direct simulations of turbulent hydromagnetic convection have provided interesting information about turbulent dynamo action. In the simulations by Nordlund et al. (1992) and Brandenburg et al. (1993) most of the dynamo activity is seen in the downdrafts. This leads to a strong downward transport of magnetic field associated with such downdrafts. This transport opposes the effects of magnetic buoyancy that tend to bring magnetic flux tubes to the surface. This is an important property, because it indicates that a dynamo may still work even in the presence of strong magnetic buoyancy. This downward transport causes an accumulation of the magnetic field at the interface between the radiative interior and the convection zone. Even though the magnetic energy density achieves its maximum at this interface, dynamo action is not restricted to this region, but occurs indeed at all depths throughout the convection zone.

The magnetic field obtained in simulations is highly disordered and the orientation of magnetic flux tubes seems to be quite random. In order to explain Hale's polarity law one might expect that one needs a strong toroidal field consisting of flux tubes oriented mainly in one and the same direction (cf. Schüssler 1987). Whilst the field in the convection zone is probably highly intermittent, it is to be expected that there is still a nonvanishing mean-field component, whose strength may well be a hundred times weaker than the rms field strength. We might expect that, as the magnetic field is pushed down to the bottom of the convection zone, the field becomes more and more uniformly oriented towards the overshoot layer. Here, the turbulence is weak and there is enough time for individual flux tubes to align themselves according to the polarity of the majority of flux tubes down there. According to this picture, flux cancellation due to fast reconnection together with shear and persistent downward pushing motions from above might be able to cause a sufficiently uniform orientation of the strongest flux tubes which eventually escape and rise to the surface to produce a sunspot pair.

The process outlined above has not yet been seen in numerical simulations, and it is plausible that such an effect only occurs if the simulation was carried out in a box large enough to allow both differential rotation and large-scale (mean-field type) dynamo action in the convection zone proper.

It is suggestive to associate the mechanism of flux tube alignment both above and below the convection zone proper with the behaviour of a compass needle in a large-scale magnetic field. If external perturbations are weak the compass needle will be able to align itself. The only possible regions where this is the case are the upper and lower overshoot layers. The typical timescale for the alignment of an idealised, "freely moving" magnetic flux tube with a field strength $B_{1}$ in an external 
magnetic field $B_{0}$ depends on the density and the length $L$ of the needle. To order of magnitude we have

$$
\tau=L / v_{A}, \quad \text { where } \quad v_{A}^{2}=B_{0} B_{1} /\left(\mu_{0} \rho\right),
$$

and $v_{A}$ is the Alfvén speed computed from the geometrical mean of the magnetic field in and outside the magnetic flux tube. Using typical values for the surface layers, $B_{0}=1 \mathrm{G}, B_{1}=1000 \mathrm{G}, \rho=10^{-6} \mathrm{~g} / \mathrm{cm}^{3}$, we obtain $v_{A} \approx 0.1 \mathrm{~km} / \mathrm{s}$, and with $L=1000 \mathrm{~km}$ we have $\tau \approx 3$ hours. This time is short enough for magnetic flux tubes to reorient themselves after their emergence. In the lower overshoot region the time scales are much longer, but still short compared with the period of the solar cycle, and so this effect may be important even down in the overshoot layer. Sufficiently large simulations including upper and lower overshoot layers could, in principle, display this effect.

\section{Determination of mean-field transport coefficients}

As long as it is impossible to run a simulation large enough to resolve simultaneously the enormous range of time and length scales to describe convective motions in a model for the 22 year solar cycle, it is obviously useful to understand basic processes using mean-field ideas. Simulations can be used to test the validity of the meanfield approach and to get independent estimates for the various mean-field transport coefficients.

\subsection{THE $\alpha$-EFFECT}

Most of the attempts to determine the $\alpha$-effect and other turbulent transport coefficients have been carried out using the First-Order-Smoothing approach. Given its limitations it is important to investigate other possibilities. One way is to measure the mean electromotive force $\left\langle\mathbf{u}^{\prime} \times \mathbf{B}^{\prime}\right\rangle$ in the presence of an imposed magnetic field $\langle\mathbf{B}\rangle$ of different strength and direction. A number of results have been obtained in this way. For example, there have been some preliminary indications that the vertical component of the $\alpha$-effect may actually have a different sign than the other two horizontal components along the diagonal of the $\alpha$-tensor (Brandenburg et al. 1990). Qualitatively similar results have recently been obtained analytically by Ferriére (1993) and Rüdiger \& Kichatinov (1993).

An important future application is the determination of the latitudinal dependence of $\alpha$, which is analytically known only for the special case where the stratificational effects on the turbulence are weak. In particular at the bottom of the convection zone the radial gradient of the turbulence intensity is larger, and therefore higher powers of terms proportional to $\mathrm{g} \cdot \Omega \propto \cos \theta$ should appear in the $\alpha$-effect (see, e.g., Rüdiger 1978).

Unfortunately, measuring the $\alpha$-effect in more realistic simulations can be quite time consuming. More turbulent runs can show quite lengthy transient behaviour and, in order to obtain good statistics and well-converged averages, the runs have to be sufficiently long. This is particularly the case when there is a dynamo effect generating significant small-scale magnetic fields. There are some indications that 
in the presence of dynamo action there is an enhanced probability of the velocity and magnetic field vectors being aligned. In this case the electromotive force is strongly reduced and it is difficult to extract a value for the $\alpha$-effect.

\subsection{QUENCHING}

As the magnetic field increases, the feedback via the Lorentz force modifies the turbulent motions and thereby the $\alpha$-effect. Again, the predictions from mean-field theory can be tested. In Fig. 2 we reproduce results obtained in Brandenburg et al. (1993) for an imposed horizontal magnetic field $B_{0}$. In the left hand panel we compare our results with the prediction of Rüdiger \& Kichatinov (1993). Note that, according to the numerical results, quenching sets in much earlier than the quenching derived from mean-field theory. Our results are in rough agreement with simulations of Tao et al. (1993).
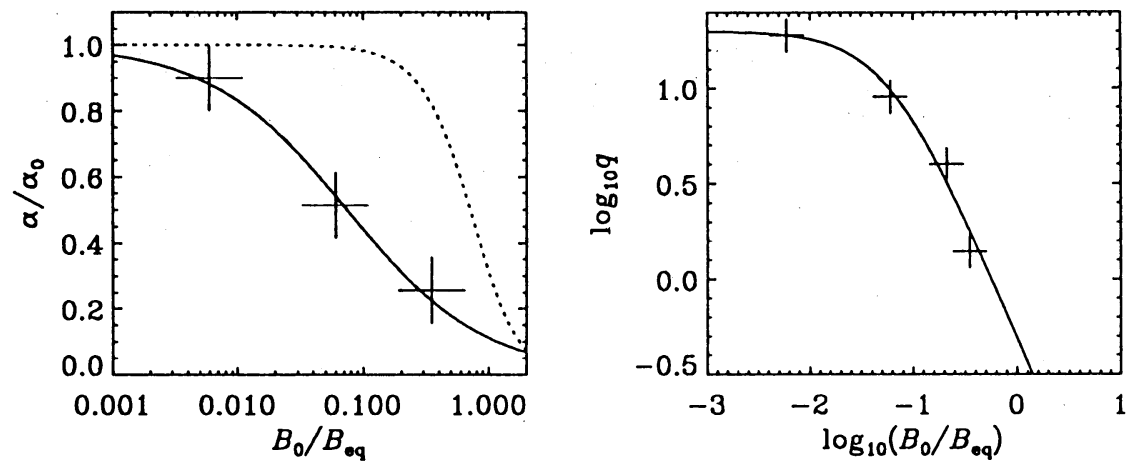

Fig. 2. Quenching of the $\alpha$-effect (left panel) and the strength of magnetic fluctuations measured in terms of $q$ (right panel), as a function of the imposed magnetic field strength $B_{0}$ (normalised by the equipartition value $B_{\mathrm{eq}}=\mu_{0} \rho u_{t}^{2}$ ). The dotted line in the left hand panel refers to the analytical result of Rüdiger \& Kichatinov (1993) for a strong uniform magnetic field.

Most of the current approaches to determine $\alpha$-quenching and other magnetic feedbacks are limited to the case where a uniform magnetic field is assumed which then modifies the turbulence. The main problem with this approach is obviously that the mean-field in the Sun is most probably not smooth, but consists of many small flux tubes. This implies that the maximum magnetic field in individual flux tubes is much larger than the mean magnetic field. The intermittent properties of the mean magnetic field may be characterised by the moments of the magnetic field, in particular by the quantities $q \equiv\left\langle\mathbf{B}^{\prime 2}\right\rangle / B_{0}^{2}$ and the kurtosis $\left\langle\mathbf{B}^{4}\right\rangle /\left\langle\mathbf{B}^{2}\right\rangle^{2}$. In the right hand panel of Fig. 2 we have plotted $q$ as a function of the imposed magnetic field strength $B_{0}$. Note that $q$ is significantly quenched as $B_{0}$ approaches the equipartition value $B_{\text {eq }}=\mu_{0} \rho u_{t}^{2}$, where $u_{t}$ is the rms velocity of the turbulent motions. A similar result has been obtained by Kleeorin et al. (1990). For further 
details see Brandenburg et al. (1993).

\subsection{THE $\Lambda$-EFFECT}

Pulkkinen et al. $(1991,1993)$ have presented an attempt to measure the components $Q_{r \phi}$ and $Q_{\theta \phi}$ of the Reynolds stress tensor from simulations carried out at different latitudes and to determine the latitudinal dependence of the $\Lambda$-effect. Furthermore, these simulations have provided first results concerning the $Q_{r \theta}$ component of the Reynolds tensor, which might be important for causing noncylindrical $\Omega$-contours in the Sun.

\subsection{THE TURBULENT BAROCLINIC TERM}

In the limit of rapid rotation and small eddy viscosity the gradient of the angular velocity in the direction along the rotation axis can only be balanced by the baroclinic term, i.e.

$$
-\varpi \partial \Omega^{2} / \partial z \approx \nabla\langle T\rangle \times \nabla\langle S\rangle+\left\langle\nabla T^{\prime} \times \nabla S^{\prime}\right\rangle
$$

where $\varpi=r \sin \theta$ is the distance from the rotation axis and $z=r \cos \theta$ the distance from the equatorial plane, $T$ is temperature and $S$ specific entropy. The term $\nabla\langle T\rangle \times \nabla\langle S\rangle$ is treated explicitly in the mean-field computations, but the turbulent contribution $\left\langle\nabla T^{\prime} \times \nabla S^{\prime}\right\rangle$, arising from the correlation between $T$ and $S$, has to be derived from a turbulence model or a simulation. In Fig. 3 we show the result for a model presented in Brandenburg et al. (1993) with a Rayleigh number $\mathrm{Ra}=10^{7}$, a Taylor number $\mathrm{Ta}=10^{6}$, and a Prandtl number $\operatorname{Pr}=0.5$.

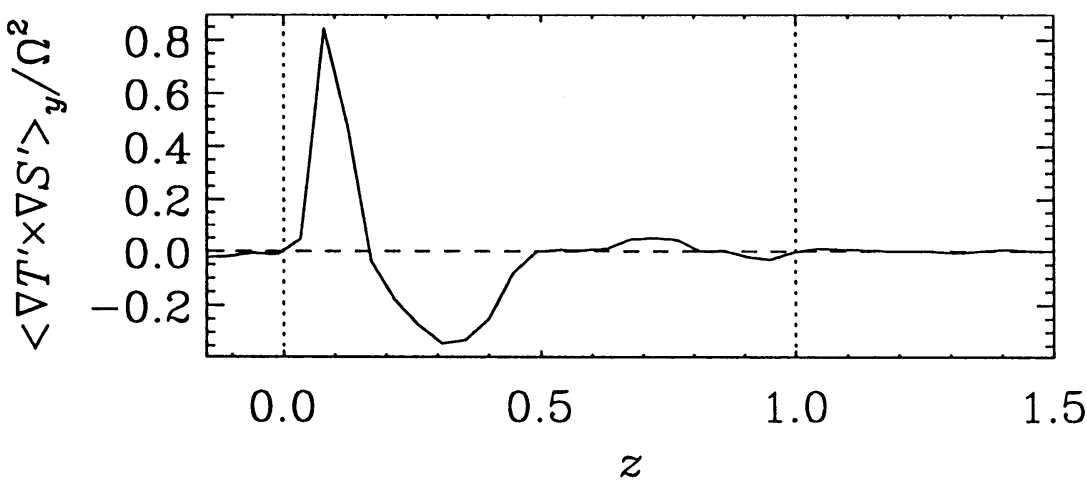

Fig. 3. The turbulent contribution to the baroclinic term, derived from a simulation. The boundaries between the upper and lower overshoot layers at $z=0$ and $z=1$, respectively, are indicated by vertical dotted lines.

Note that the turbulent contribution to the baroclinic term becomes large only close to the surface of the domain, and is negligible in the lower parts. Thus, sub- 
stantial deviations from cylindrical $\Omega$-contours can only be expected in the upper part of the convection zone.

\section{Conclusions}

The computation of solar mean-field dynamo models encounters several problems. It is well-known that $\alpha \Omega$-type dynamos can reproduce the solar cycle period only if the turbulent magnetic diffusivity is much smaller than the standard value. On the other hand, there are arguments in favour of relatively large values of the eddy viscosity and the eddy conductivity. However, the possibility of strong viscous heating puts an upper limit on the value of the eddy viscosity. Thus, we argue that $\eta_{t} \ll \nu_{t} \ll \chi_{t}$. This property might be related to the different degrees of intermittency of the magnetic, velocity, and entropy fields. In this connection it is interesting to note that Childress (1979) found for simple steady flows that the magnetic field is highly intermittent and $\alpha$ is only of the order of the square of the magnetic Reynolds number; see also Perkins \& Zweibel (1987). For turbulent flows the reduction may be less extreme, but qualitatively similar arguments may also apply to the reduction of the turbulent magnetic diffusivity when the magnetic field is intermittent; see the reviews of Schüssler (1983) and Gilman (1986).

It remains a challenging task to set up a good direct simulation that produces differential rotation and magnetic fields similar to those observed in the Sun. Of particular interest is the question of how Hale's polarity law can be explained. In the picture favoured in this paper a weak mean magnetic field is generated in the entire. convection zone. There the actual magnetic field is highly intermittent and much larger than the mean magnetic field. Turbulent pumping acts against magnetic buoyancy and causes the field to accumulate at the interface between convection zone and radiative interior. Here, the time scales are long and reconnection processes can cause the cancellation of flux tubes with opposite polarity. The result is a more uniformly oriented bundle of flux tubes that can be amplified further by the action of differential rotation. It is desirable to see the pieces of this picture being realised in simulations. Another mechanism that needs to be investigated in a direct simulation is the subsequent realignment of flux tubes once they have reached the surface.

\section{Acknowledgements}

I thank the Isaac Newton Institute and the members of the Dynamo Theory group for a very stimulating atmosphere. I am grateful to David Moss, Åke Nordlund, Günther Rüdiger, Ed Spiegel, Ilkka Tuominen, Nigel Weiss, and Ellen Zweibel for comments and stimulating discussions. This work was supported in part by SERC.

\section{References}

Brandenburg, A., Nordlund, A., Pulkkinen, P., Stein, R.F., Tuominen, I.: 1990, Astron. Astrophys. 232, 277

Brandenburg, A., Moss, D., Tuominen, I.: 1992a, in The Solar Cycle, ed(s)., K. L. Harvey, ASP Conference Series, Vol. 27, 536

Brandenburg, A., Moss, D., Tuominen, I.: 1992b, Astron. Astrophys. 265, 328 
Brandenburg, A., Krause, F., Nordlund, Å., Ruzmaikin, A. A., Stein, R.F., Tuominen, I.: 1993, Astrophys. J. (submitted)

Brown, T. M., Morrow, C. A.: 1987, Astrophys. J. 314, L21

Childress, S.: 1979, Phys. Earth Planet. Int. 20, 172

Choudhuri, A. R.: 1990, Astrophys. J. 355, 733

Durney, B. R., Spruit, H. C.: 1979, Astrophys. J. 234, 1067

Durney, B. R.: 1987, in The internal solar angular velocity, ed(s)., B. R. Durney E S. Sofia, Reidel, Dordrecht, 235

Ferrière, K.: 1993, Astrophys. J. 404, 162

Gilman, P. A.: 1983, Astrophys. J. Suppl. 53, 243

Gilman, P. A.: 1986, in Physics of the Sun. Vol. I: The solar interior, ed(s)., P. A. Sturrock, Reidel, Dordrecht, 95

Gilman, P. A.: 1992, in The Solar Cycle, ed(s)., K. L. Harvey, ASP Conference Series, Vol. 27, 241

Gilman, P. A., Miller, J.: 1981, Astrophys. J. Suppl. 46, 211

Gough, D.O., Kosovichev, A.G., Sekii, T., Libbrecht, K.G., Woodard, M.F.: 1992, The form of the angular velocity in the solar convection zone (preprint)

Glatzmaier, G. A.: 1985a, Astrophys. J. 291, 300

Glatzmaier, G. A.: 1985b, Geophys. Astrophys. Fluid Dyn. 31, 137

Glatzmaier, G. A., Gilman, P. A.: 1982, Astrophys. J. 256, 316

Hewitt, J. M., McKenzie, D. P., Weiss, N. O.: 1975, J. Fluid Mech. 68, 721

Kichatinov, L. L.: 1991, Astron. Astrophys. 243, 483

Kleeorin, N. I., Rogachevskii, I. V., Ruzmaikin, A. A.: 1990, Sov. Phys. JETP 70, 878

Krause, F., Rädler, K.-H.: 1980, Mean-Field Magnetohydrodynamics and Dynamo Theory, Akademie-Verlag, Berlin

Libbrecht, K. G.: 1988, in Proc. Symp. Seismology of the Sun and Sun-like Stars, ed(s)., E. J. Rolfe, ESA SP-286, 131

Meneguzzi, M., Pouquet, A.: 1989, J. Fluid Mech. 205, 297

Nordlund, A.: 1985, Solar Phys. 100, 209

Nordlund, A., Brandenburg, A., Jennings, R. L., Rieutord, M., Ruokolainen, J., Stein, R. F., Tuominen, I.: 1992, Astrophys. J. 392, 647

Parker, E. N.: 1993, Astrophys. J. (submitted)

Perkins, F. W., Zweibel, E. G.: 1987, Phys. Fluids 30, 1079

Pulkkinen, P., Tuominen, I., Brandenburg, A., Nordlund, §., Stein, R.F.: 1991, in The Sun and cool stars: activity, magnetism, dynamos, ed(s)., I. Tuominen, D. Moss \& G. Rüdiger, Lecture Notes in Physics 380, Springer-Verlag, 98

Pulkkinen, P., Tuominen, I., Brandenburg, A., Nordlund, A., Stein, R.F.: 1993, Astron. Astrophys. 267, 265

Roberts, P. H., Stix, M.: 1972, Astron. Astrophys. 18, 453

Rüdiger, G.: 1978, Astron. Nachr. 299, 217

Rüdiger, G.: 1987, Acta Astron. 37, 223

Rüdiger, G., Kichatinov, L. L.: 1993, Astron. Astrophys. (in press)

Rüdiger, G., Tuominen, I.: 1990, in Solar Photosphere: Structure, Convection and Magnetic Fields, ed(s)., J. O. Stenflo, Kluwer Acad. Publ., Dordrecht, 315

Schmitt, D.: 1993 (these Proceedings)

Schüssler, M.: 1983, in Solar and stellar magnetic fields, ed(s)., J. O. Stenflo, Reidel, 213

Schüssler, M.: 1987, in The internal solar angular velocity, ed(s)., B. R. Durney $\&$ S. Sofia, Reidel, Dordrecht, 303

Spruit, H. C.: 1977, Astron. Astrophys. 55, 151

Steenbeck, M., Krause, F.: 1969, Astron. Nachr. 291, 49

Stein, R.F., Nordlund, A.: 1989, Astrophys. J. 342, L95

Tao, L., Cattaneo, F., Vainshtein, S. I.: 1993, in Theory of Solar and Planetary Dynamos, ed(s)., P.C. Matthews \& A.M. Rucklidge, Cambridge University Press, (in press)

Tuominen, I., Brandenburg, A., Moss, D., Rieutord, M.: 1993, Astron. Astrophys. (in prep.)

Vainshtein, S. I.: 1972, Sov. Phys. JETP 34, 327

Vainshtein, S. I., Cattaneo, F.: 1992, Astrophys. J. 393, 165 